Psychother Psychosom 2018;87:121-123

DOI: $10.1159 / 000486793$

\section{Symptom-Specific Effects of Psychotherapy versus Combined Therapy in the Treatment of Mild to Moderate Depression: A Network Approach}

\author{
Ella Bekhuis ${ }^{a}$ Robert Schoevers ${ }^{a}$ Marrit de Boer ${ }^{a}$ Jaap Peen ${ }^{b}$ \\ Jack Dekker ${ }^{b}$ Henricus Van ${ }^{b}$ Lynn Boschloo ${ }^{a}$

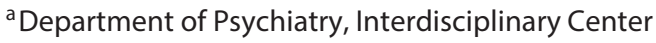 \\ Psychopathology and Emotion Regulation (ICPE), University \\ Medical Center Groningen, University of Groningen, Groningen, \\ The Netherlands; ${ }^{b}$ Research Department, Arkin Mental Health \\ Care, Amsterdam, The Netherlands
}

A number of studies have reported that adding pharmacotherapy to psychotherapy has no or only small advantages in the treatment of mild to moderate depression [1-3]. These studies have used sum scores of depression rating scales as effect parameters [1-3]. However, as individual items on these scales have recently been shown to respond differentially to pharmacotherapy compared to placebo [4], the effects of an addition of pharmacotherapy to psychotherapy may only be detectable by focusing on individual depressive symptoms.

Previous studies investigating treatment responses of individual depressive symptoms (e.g., Hieronymus et al. [4]) did not take into account the potential interrelatedness of these symptoms. For example, patients who become less self-blaming in response to treatment may also be more likely to experience reductions in feelings of worthlessness or blue mood. Tools to consider symptom interrelatedness are offered by the network approach, which conceptualizes depression as a system of associated symptoms [5]. Earlier network studies have demonstrated that depressive symptoms are differentially related to one another [5-7]; however, it remains unknown if similar association patterns exist among changes in these symptoms during treatment. Taking into account these relations in a network structure provides the opportunity to determine the effects of adjunctive pharmacotherapy on specific symptoms while adjusting for responses of other symptoms. This enables a differentiation between direct symptom-specific effects (i.e., those independent of changes in other symptoms) and indirect symptom-specific effects (i.e., those mediated by changes in other symptoms).

This is the first study to determine the relative efficacy of psychotherapy versus combined therapy on individual depressive symptoms. Data were derived from a randomized controlled trial comparing short-term psychodynamic supportive psychotherapy (SPSP) and this therapy combined with pharmacotherapy in patients with mild to moderate depression [1]. Participants consisted of newly registered patients at 2 outpatient facilities in Amsterdam (The Netherlands) of age 18-65 years with a DSM-IV-defined major depressive disorder of mild to moderate severity. SPSP involves an open patient-therapist dialogue that uses supportive and insight-facilitating techniques to address the emotional background of depression and was delivered in 16 sessions of 45 min within a 24 -week period. In the combined condition, antidepressants were provided for 24 weeks according to a protocol with several steps in case of intolerance or inefficacy: first venlafaxine, followed by fluoxetine, and finally nortriptyline. Sixteen depressive symptoms were assessed at baseline and after 24 weeks with the depression subscale of the Symptom Checklist-90. Analyses were conducted in a sample consisting of all patients who started with the treatment they were allotted to (psychotherapy, $n=103$; combined therapy, $n=83$; see the online suppl. material for the sample characteristics; for all online suppl. material, see www.karger.com/doi/10.1159/000486793) and the last outcome carried forward method was applied. First, we focused on the relative efficacy of psychotherapy versus combined therapy by using individual symptoms as effect parameters and, then, differentiated between direct and indirect effects by taking into account symptom interrelatedness in a network model.

Symptom-specific efficacy of psychotherapy versus combined therapy was investigated using independent sample $t$ tests with change scores (post- minus pre-treatment) of depressive symptoms as dependent variables. Combined therapy was significantly more effective than psychotherapy in decreasing the symptoms feeling entrapped (Cohen's $d=0.55, p<0.001$ ), emotional lability (Cohen's $d=0.47, p=0.002$ ), worry (Cohen's $d=0.44, p=0.003$ ), hopelessness (Cohen's $d=0.41, p=0.006$ ), obsessive thoughts (Cohen's $d=0.34, p=0.02$ ), blue mood (Cohen's $d=0.32, p=0.03$ ), and low in energy (Cohen's $d=0.31, p=0.04$ ). The remaining 9 symptoms showed similar responses to psychotherapy and combined therapy (Fig. 1).

Then, we took into account symptom interrelatedness to differentiate between the direct and indirect effects of the addition of pharmacotherapy to psychotherapy. An L1-regularized partial correlation network of treatment type and change scores of all depressive symptoms was estimated (the network estimation procedure and tests for parameter estimate accuracy are described in the online suppl. material). Figure 1 shows that changes in depressive symptoms during treatment were strongly related. The strongest association was found between thoughts of death and thoughts of suicide (partial correlation $=0.49$ ), indicating that persons with an improvement in thoughts of death during treatment were more likely to experience an improvement in thoughts of suicide as well. Treatment type showed the strongest direct connections to feeling entrapped (partial correlation $=0.16$ ) and emotional lability (partial correlation $=0.11$ ), and was weakly connected to worry (partial correlation $=0.04)$, low in energy (partial correlation $=0.01)$, and hopelessness (partial correlation $=0.01)$. All connections were in

\section{KARGER}

E-Mail karger@karger.com www.karger.com/pps
2018 The Author(s)

Published by S. Karger AG, Basel

Karger Open access

This article is licensed under the Creative Commons AttributionNonCommercial-NoDerivatives 4.0 International License (CC BY NC-ND) (http://www.karger.com/Services/OpenAccessLicense). Usage and distribution for commercial purposes as well as any distribution of modified material requires written permission.
Ella Bekhuis

Department of Psychiatry, University Medical Center Groningen PO Box 30.001

NL-9700 RB Groningen (The Netherlands)

E-Mail e.bekhuis@ umcg.nl 
Fig. 1. Symptom-specific effects of psychotherapy versus combined therapy. The type of treatment is represented by the square and depressive symptoms by circles. Relative effect sizes of psychotherapy versus combined therapy on specific symptoms (all in favor of combined therapy) are indicated by the size of circles and their level of significance by circle color (violet is significant and white is nonsignificant; colors online version only). Connections in the network model are represented by lines, of which the thickness is proportional to the strength of associations. Direct associations between the type of treatment and change scores of symptoms are all in favor of combined therapy (indicated by violet lines) and associations between change scores of symptoms are all positive (indicated by green lines).

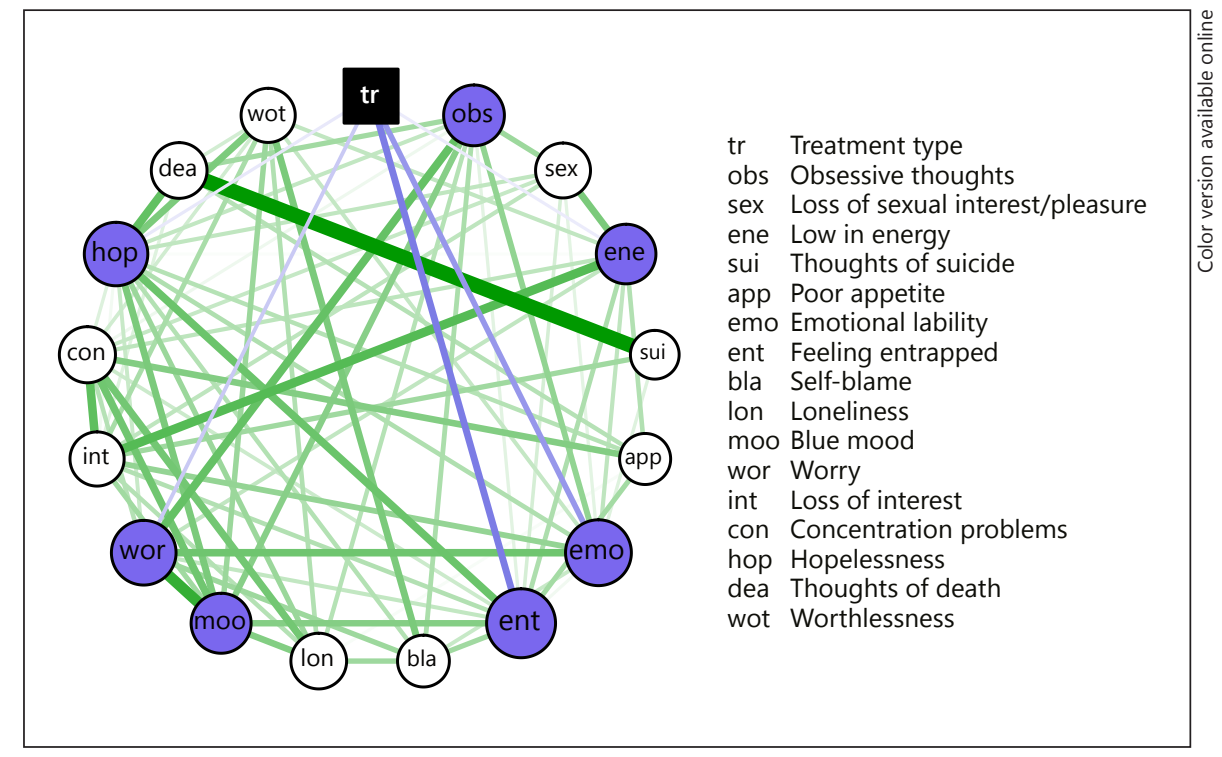

favor of combined therapy, suggesting that this therapy targeted these particular symptoms directly.

Despite their significant responses to the addition of pharmacotherapy to psychotherapy in our first analysis, obsessive thoughts and blue mood were not directly connected to treatment type in the network, and worry, low in energy, and hopelessness showed only weak direct associations to this variable. Interestingly, the network revealed that these symptoms were related to changes in feeling entrapped and emotional lability, which in turn were more strongly connected to the type of treatment. This suggests that the effect of adjunctive pharmacotherapy on obsessive thoughts, blue mood, worry, low in energy, and hopelessness may largely have been indirect and could have been mediated by changes in feeling entrapped and emotional lability.

A strength of this study is that the trial included a fairly random and representative sample of mildly to moderately depressed patients in secondary care. Furthermore, we estimated the network structure using 11-regularization to prevent overfitting, which has been shown to adequately control for false positive associations. However, in our relatively small sample of 186 individuals, small true positive associations could have been overlooked [8]. As baseline scores in our sample differed across symptoms, it is also important to note that a higher baseline severity of symptoms was associated with stronger responses to adjunctive pharmacotherapy, which is in line with previous reports [2].

In conclusion, this study showed that combined therapy outperformed psychotherapy in the treatment of some depressive symptoms and not others. Although our results are exploratory rather than conclusive, they suggest that adjunctive pharmacotherapy targeted specific symptoms (e.g., feeling entrapped, emotional lability) directly and other symptoms (e.g., obsessive thoughts, blue mood) indirectly. As direct effects are independent of changes in other symptoms, our findings imply that adjunctive pharmacotherapy can effectuate improvements in directly targeted symptoms in all patients irrespective of changes in other symptoms. Indirectly targeted symptoms, in contrast, may respond to an addition of pharmacotherapy to psychotherapy, but only in patients improving on symptoms mediating these responses during treatment and, therefore, reporting these symptoms before treatment. If replicated, these insights may help clinicians to predict which patients could benefit from an addition of pharmacotherapy to psychotherapy [9].

Given the differential treatment responses across symptoms, we would like to encourage other researchers to analyze individual depressive symptoms as well as their interrelatedness. Network models are highly promising in this approach as they can be expanded with other psychiatric or physical symptoms (e.g., anxiety, nausea) to provide insight into secondary or side effects of a treatment independent of its effects on depressive symptoms. Furthermore, dynamic networks of depressive symptoms during various treatment stages could reveal that changes in specific symptoms are preceded by changes in other symptoms, which may inform on pathways underlying indirect responses of symptoms to a treatment [10].

\section{Disclosure Statement}

The original trial was supported by an unrestricted educational grant from Wyeth Nederland.

\section{References}

1 de Jonghe F, Hendricksen M, van Aalst G, Kool S, Peen V, Van R, van den Eijnden E, Dekker J: Psychotherapy alone and combined with pharmacotherapy in the treatment of depression. Br J Psychiatry 2004;185: $37-45$.

2 de Maat SM, Dekker J, Schoevers RA, de Jonghe F: Relative efficacy of psychotherapy and combined therapy in the treatment of depression: a meta-analysis. Eur Psychiatry 2007;22:1-8.

3 Cuijpers P, van Straten A, Warmerdam L, Andersson G: Psychotherapy versus the combination of psychotherapy and pharmacotherapy in the treatment of depression: a meta-analysis. Depress Anxiety 2009;26:279_ 288. 
4 Hieronymus F, Emilsson JF, Nilsson S, Eriksson E: Consistent superiority of selective serotonin reuptake inhibitors over placebo in reducing depressed mood in patients with major depression. Mol Psychiatry 2016; 21:523-530

5 Fried EI, van Borkulo CD, Cramer AO, Boschloo L, Schoevers RA, Borsboom D: Mental disorders as networks of problems: a review of recent insights. Soc Psychiatry Psychiatr Epidemiol 2017;52:1-10.

6 Bekhuis E, Schoevers RA, van Borkulo CD, Rosmalen JG, Boschloo L: The network structure of major depressive disorder, generalized anxiety disorder and somatic symptomatology. Psychol Med 2016;46:29892998.
7 Boschloo L, van Borkulo CD, Borsboom D, Schoevers RA: A prospective study on how symptoms in a network predict the onset of depression. Psychother Psychosom 2016;85:183-184.

8 van Borkulo CD, Borsboom D, Epskamp S, Blanken TF, Boschloo L, Schoevers RA, Waldorp LJ: A new method for constructing networks from binary data. Sci Rep 2014;4:5918.

9 Dekker J, Van HL, Hendriksen M, Koelen J, Schoevers RA, Kool S, van Aalst G, Peen J: What is the best sequential treatment strategy in the treatment of depression? Adding pharmacotherapy to psychotherapy or vice versa? Psychother Psychosom 2013;82:89-98.

10 Hayes AM, Yasinski C, Barnes JB, Bockting CL: Network destabilization and transition in depression: new methods for studying the dynamics of therapeutic change. Clin Psychol Rev 2015;41:27-39. 